\title{
Stimulus frequency influences spontaneous perceptual reversals in ambiguous apparent motion
}

\author{
Rüdiger Ilg, Stefan BuraZanis, Afra M. WOHLSChläger, \\ Andreas Wöller, Stefan Wagenpfeil, and Mark Mühlau \\ Technische Universität München, München, Germany
}

\begin{abstract}
The temporal integration of continuous sensory information into a temporally extended percept becomes evident in spontaneous reversals of ambiguous apparent motion. To study the temporal relation of spontaneous percept reversals and temporal stimulus properties, we systematically varied presentation frequency in an ambiguous-apparent-motion paradigm. Moreover, we triggered percept reversals in a manner that was not consciously perceived by manipulating the duration of single frames. We found that the reversal rate depended on the stimulus frequency (with higher frequencies resulting in faster percept reversals) and that we could externally trigger perceptual reversals. Our findings support the idea that spontaneous reversals of ambiguous apparent motion are influenced by bottom-up effects at early processing levels. The paradigm allows for specific contrasts of spontaneous and externally triggered (but otherwise identical) perceptual reversals and, by this means, for further study of the underlying mechanisms.
\end{abstract}

Since the early 18 th century, reversible figures have drawn the attention of researchers who considered them a unique window for studying the underlying processes of visual perception. In the past 170 years, different kinds of reversible (or ambiguous) figures have been developed and studied using psychophysical and neurophysiological methods. Long and Toppino (2004) suggested differentiating between the ambiguity of a figure, which refers to the basic fact that the same physical stimulus can produce more than a single cognitive interpretation or percept, and the reversibility of the percept (i.e., involuntary oscillations between two percepts), which is assumed to depend on competing internal representations. In line with their proposal, our study will focus on the temporal properties of reversals in ambiguous apparent motion.

The extents to which the observed perceptual reversal of reversible figures emerges from early processes at lower levels of the visual system or results from influences of higher cognitive processes is a topic of continuing debate. In extensive reviews of the phenomenon of perceptual ambiguity in reversible figures, Leopold and Logothetis (1999) and Long and Toppino (2004) summarized the proposed models of "figural reversals." By and large, theories on reversible figures are based on either bottomup or top-down classes of explanations. Behavioral data lend support to both of these classes.

The first set of explanations assumes that perceptual reversal is primarily based on a rivalry between two competing perceptual interpretations at early processing levels - that is, between the retina and the corresponding receptive fields of the primary visual cortex (Blake, Sobel, \& Gilroy, 2003). Indeed, the reversal of figures depends on stimulus properties such as the intensity (Lynn, 1961), figural completeness (Babich \& Standing, 1981), and continuity of presentation (Leopold, Wilke, Maier, \& Logothetis, 2002; Orbach, Ehrlich, \& Heath, 1963). Further results that are typically cited to support such a sensory, bottom-up model of figural reversal are the increasing number of reversals over time, the strong retinotopy of the reversal effect (Blake et al., 2003; Toppino \& Long, 1987), the reversebias and adaptation effects (Long \& Olszweski, 1999; Orbach et al., 1963; Petersik, Shepard, \& Malsch, 1984), and the fact that independent reversals occur when simultaneously viewing multiple figures (Babich \& Standing, 1981; Toppino \& Long, 1987).

In contrast, top-down explanations emphasize the influence of higher cognitive processes. This interpretation is supported by the observation of a certain degree of volitional control and by electrophysiological evidence (Leopold \& Logothetis, 1999). First and foremost, voluntary and intentional control is cited as an expression of topdown influences (Hol, Koene, \& van Ee, 2003; Peterson \& Hochberg, 1983; Toppino, 2003). Slotnick and Yantis (2005) showed that voluntary shifts of visual attention and the perceptual configuration of a "Necker-cube-like" stimulus (composed of moving dots) were associated with activity in the posterior parietal cortex and frontoparietal attentional control network that has been interpreted to

R. Ilg, ilg@neuro.med.tum.de 
support the hypothesis that voluntary shifts of bistable percepts are mediated by spatial attention. Further results typically cited to support such a cognitive, top-down model of figural reversal refer to the influence of attention effects (Hochberg \& Peterson, 1987; Rock, Hall, \& Davis, 1994; Toppino, 2003), to familiarity effects (Rock et al., 1994; Rock \& Mitchener, 1992), to learning and practice effects (Beer, 1989; Long, Toppino, \& Kostenbauder, 1983), to expectancy or set effects (Bruner, Busiek, \& Minturn, 1952; Bugelski \& Alampay, 1961; Long, Toppino, \& Mondin, 1992), or to the influence of mental workload (Reisberg, 1983; Reisberg \& O'Shaughnessy, 1984).

Also, both top-down and bottom-up influences on spontaneous reversals of bistable percepts are supported by electrophysiological evidence, which is somehow contradictory. Electrophysiological recordings of binocular rivalry in monkey brains have shown that reversals were primarily associated with activation in higher extrastriate visual areas and that monkeys' subjective perceptions corresponded most to the activity of visually responsive neurons in the inferotemporal cortex and superior temporal sulcus (Logothetis \& Schall, 1989). The observation that perceptual rivalry was associated with activation in later visual areas has been interpreted to support a top-down initiation. Results of human fMRI experiments on binocular rivalry and ambiguous figures point in the same direction (Kleinschmidt, Büchel, Zeki, \& Frackowiak, 1998; Lumer, Friston, \& Rees, 1998; Lumer \& Rees, 1999). On the basis of such evidence for the influence of higher cortical areas, Leopold and Logothetis (1999) concluded that different perceptions of ambiguous stimuli are essentially "steered and modified by central brain structures involved in planning and generating behavioral actions" (p. 254). In a later study, Leopold et al. (2002) showed that spontaneous reversals of multistable percepts can be slowed when stimuli are viewed intermittently rather than continuously, which challenges the notion that perceptual reversals are governed by an autonomous oscillator that operates independently of the visual stimulus. Contrary to their earlier considerations, they interpreted this finding as an expression of bottom-up influences. With the help of motion-induced blindness, they further showed that the stabilization effect depended on the subjective disappearance of the pattern rather than on the disappearance of the sensory representation itself. Perceptual switching thus appeared to be associated with mechanisms on the perceptual rather than the sensory processing level. Reviewing the evidence on binocular rivalry and ambiguous figures, both Blake and Logothetis (2002) and Long and Toppino (2004) came to the conclusion that bistable perception relies on an interaction of sensory and cognitive processes, including bottom-up and top-down mechanisms.

As outlined above, the earlier studies indicated that bistable perception is influenced by stimulus parameters such as the visual angle (Borsellino et al., 1982) as well as the speed and density of presentation (Brouwer \& van Ee, 2006). Furthermore, perceptual alternations can be triggered by a transient stimulus presented near the perceived object (Kanai, Moradi, Shimojo, \& Verstraten, 2005). Following Brouwer and van Ee's approach, we modified an ambiguous-apparent-motion paradigm that allowed us to further study the temporal constraints of spontaneous perceptual reversals by systematically varying the stimulus frequency and duration of single frames. We studied the relation between the frequency of perceptual reversals and the stimulus frequency (Experiment 1), as well as whether a perceptual reversal can be triggered by a small irregularity in the presentation sequence (Experiment 2). A further aim of Experiment 2 was to develop a paradigm that would allow us to externally trigger otherwise spontaneous perceptual reversals. Since we were focusing on the temporal properties of spontaneous perceptual reversals, we chose a "simple" apparent-motion paradigm in which identical percepts were induced (apart from the rotation direction), thus enabling us to rule out higher cognitive influences. Such influences as secondary shifts of visuospatial attention and other effects secondary to the changing perceptual content are common confounding variables in paradigms using complex ambiguous pictures.

\section{METHOD}

\section{Subjects}

Twenty-seven volunteer subjects ( 18 males, 9 females; mean age 27 years, range $20-36$ years) with normal or corrected-to-normal vision participated in the study. All were right-handed and drug-free and had no history of neurological or psychiatric disorders, and all gave written informed consent before the experiments. The study was approved by the Ethics Committee of the Faculty of Medicine at Technische Universität München.

\section{Stimulus Materials and Task}

The stimulus consisted of a modified version of the stimulus that Wohlschläger (2000) used in an apparent-motion paradigm to study the influence of action on the perception of apparent visual motion. This kind of illusory-motion stimulus dates from the spinning-wheel illusion first described by Wertheimer (1912) and later used in varied versions, mainly focusing on the perception of motion (Sterzer \& Kleinschmidt, 2007; Sterzer, Russ, Preibisch, \& Kleinschmidt, 2002; for a review of related studies, see Wilms et al., 2005) and on the influence of such factors as attention (Cavanagh, 1992; Culham, Verstraten, Ashida, \& Cavanagh, 2000; Verstraten \& Ashida, 2005; Verstraten, Cavanagh, \& Labianca, 2000). In our experiments, we displayed a circle of 15 black dots on a gray background with a central fixation cross (we will refer to this as a "frame"). Two identical frames were alternately displayed, with an offset angle of $12^{\circ}$, thus inducing the impression of a circle that ambiguously rotated either in one direction or the other (Figure 1A). The stimuli were presented on a computer screen using Presentation software (Version 9.2001.28.05, Neurobehavioral Systems), at a distance of $60 \mathrm{~cm}$ from the subject's eyes. The stimulus frequency (in frames per second, or fps) and the duration of a single frame were systematically varied. Subjects were not informed about the illusory, ambiguous, and manipulative character of the task but were simply instructed to maintain fixation on the central fixation cross and to indicate changes in the rotation direction of the circle through a buttonpress with the right index finger. Trials were terminated by this response and were followed by a randomized interval of 1 or $2 \mathrm{sec}$. If a subject did not perceive a change of direction or did not respond, the trial stopped automatically after $10 \mathrm{sec}$.

We conducted two experiments. In Experiment 1, our focus was the influence of the stimulus frequency on the percept duration and spontaneous reversal rate. We therefore systematically varied the frequency (i.e., the perceived "speed" of the rotation). The experiment consisted of three runs, comprising 30 trials each. Three types of trials, with different stimulus frequencies $(7.5,6.0$, or $5.0 \mathrm{fps})$, 
A

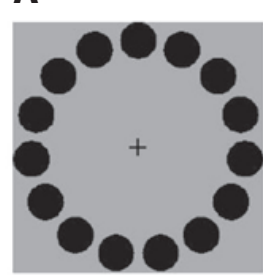

$0^{\circ}$

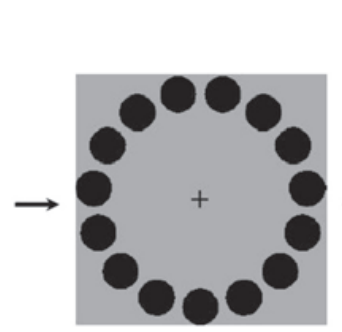

$12^{\circ}$

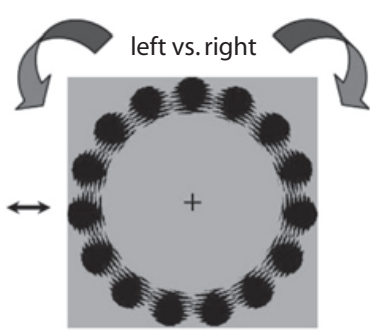

Rotation

B

1) Internally generated
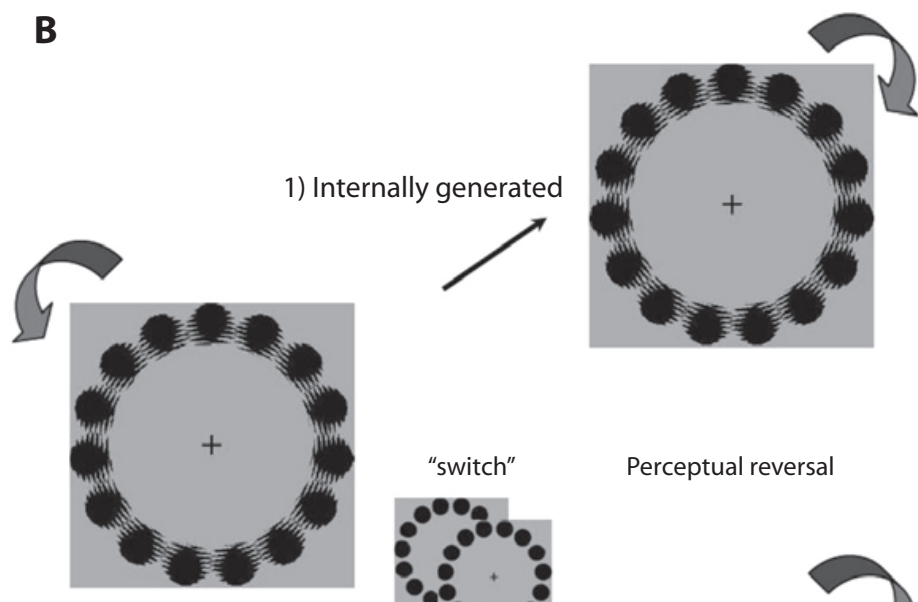

"switch"

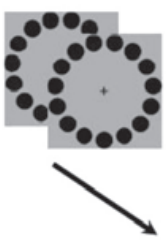

2) Externally triggered

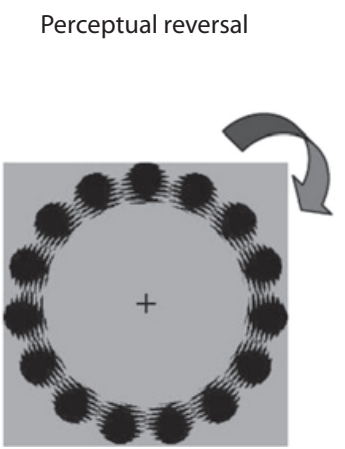

Figure 1.Ambiguous-apparent-motion stimulus (A) and experimental conditions (B).

were displayed in random order ( 90 trials overall) in order to control for adaptation effects related to the course of the experiment. The runs were separated by short breaks $(1 \mathrm{~min})$ that allowed the subjects to close their eyes and relax.

In Experiment 2, we studied whether we could trigger a perceptual reversal by introducing an irregularity in the presentation frequency, with the aim of developing a paradigm that would allow for comparisons of externally triggered with spontaneous (but otherwise identical) perceptual reversals. On the basis of the observation that spontaneously generated reversals were accompanied by the impression of a remarkable standstill, before the circle rotated in the opposite direction, we systematically prolonged the duration of single stimulus frames (a "switch" event) by either $50 \%$ or $100 \%$ in order to externally trigger a perceived reversal in the motion direction. The switch events were randomly inserted 1-2 sec after stimulus onset - that is, before the mean spontaneous reversal (which was estimated from a preliminary study) - to allow for a statistical differentiation of spontaneous responses (i.e., those internally generated by the brain) and externally triggered responses (i.e., those generated by the experimenter) (see Figure 1B). If a subject responded before a "switch" occurred, the change of rotation direction was counted as the result of a spontaneous perceptual reversal, whereas all perceptual reversals that occurred after a switch were counted as externally triggered. The experiments took about $45 \mathrm{~min}$, which was the upper limit of maintaining concentration and focus on the stimulus. The left side of Table 1 summarizes the experimental conditions.

\section{Statistical Analysis}

The single trials and the different conditions were analyzed using statistical tests for dependent samples. Since the data were not normally distributed, only nonparametric tests were applied (Friedman two-way ANOVA, Wilcoxon matched-pairs signed rank test, and Spearman's rank correlation coefficient). All $p$ values were twosided and based on a significance level of .05 .

\section{RESULTS}

\section{Baseline Parameters}

The following results include all answers of all of the subjects. In 39 of the 4,860 trials $(0.8 \% ; 23$ trials in Experiment 1 and 16 trials in Experiment 2), subjects did not respond within $10 \mathrm{sec}$, either indicating that no perceptual reversal was observed or that the subject forgot to respond. None of the subjects realized the underlying experimental design and different conditions. 
Table 1

Experimental Conditions and Percept Durations

\begin{tabular}{|c|c|c|c|c|c|c|c|c|c|}
\hline \multirow[b]{2}{*}{ Run $^{*}$} & \multirow[b]{2}{*}{ Trials } & \multirow{2}{*}{$\begin{array}{l}\text { Stimulus } \\
\text { Frequency } \\
\text { (fps) }\end{array}$} & \multirow{2}{*}{$\begin{array}{c}\text { Frame } \\
\text { Duration } \\
(\mathrm{msec})\end{array}$} & \multirow[b]{2}{*}{ "Switch" } & \multirow{2}{*}{$\begin{array}{c}\text { Total } \\
\text { Trials } \\
\end{array}$} & \multicolumn{3}{|c|}{ Percept Duration (sec) } & \multirow[b]{2}{*}{$p$} \\
\hline & & & & & & Mean & Median & Interquartile & \\
\hline \multicolumn{10}{|c|}{ Experiment 1 (No Switch) } \\
\hline 1 & 30 & 7.5 & 133 & $\mathrm{n} / \mathrm{a}$ & 799 & 3.09 & 2.58 & 1.90 & $<.001^{* *}$ \\
\hline 2 & 30 & 6.0 & 167 & $\mathrm{n} / \mathrm{a}$ & 802 & 3.12 & 2.68 & 1.94 & \\
\hline 3 & 30 & 5.0 & 200 & $\mathrm{n} / \mathrm{a}$ & 806 & 3.38 & 2.85 & 2.18 & \\
\hline \multicolumn{10}{|c|}{ Experiment 2 (Switch) } \\
\hline 1 & 30 & 7.5 & 133 & $+50 \%(67 \mathrm{msec})$ & 371 & 3.07 & 2.47 & 1.75 & $.980^{* * *}$ \\
\hline \multirow[t]{2}{*}{2} & 30 & 6.0 & 167 & $+50 \%(84 \mathrm{msec})$ & 360 & 2.90 & 2.35 & 1.58 & $.345^{* * *}$ \\
\hline & & & & $+100 \%(167 \mathrm{msec})$ & 343 & 2.78 & 2.30 & 1.16 & \\
\hline \multirow[t]{2}{*}{3} & 30 & 5.0 & 200 & $+50 \%(100 \mathrm{msec})$ & 344 & 3.08 & 2.48 & 1.89 & $.001^{* * *}$ \\
\hline & & & & $+100 \%(200 \mathrm{msec})$ & 332 & 2.79 & 2.27 & 1.46 & \\
\hline
\end{tabular}

${ }^{*}$ All runs and trials were randomized. ${ }^{* *}$ Spearman. ${ }^{* * *}$ Friedman.

\section{Correlation of Percept Duration With Stimulus Frequency}

In Experiment 1 (see Table 1, upper panel), we studied the influence of the stimulus frequency on the percept duration. The median percept duration (from stimulus onset to buttonpress) of all stimuli was $2.69 \mathrm{sec}$. Different stimulus frequencies resulted in significantly different percept durations (Spearman $p<.001$ ), in that higher frequencies resulted in shorter durations $(2.58 \mathrm{sec}$ at $7.5 \mathrm{fps}, 2.68 \mathrm{sec}$ at $6.0 \mathrm{fps}$, and $2.85 \mathrm{sec}$ at $5.0 \mathrm{fps}$ ). Converted to frames, the median percept durations were 19.4 frames in the 7.5 -fps condition, 16.1 frames in the 6-fps condition, and 14.25 frames in the 5 -fps condition. Pairwise comparisons revealed significant differences in percept duration between the 6- and 5-fps conditions and between the 7.5- and 5-fps conditions (Wilcoxon $p<.001$ ), but no significant difference was found between the 7.5- and 6-fps conditions (Wilcoxon $p=.25$ ).

\section{Effect of the Manipulation of the Percept Reversal}

In Experiment 2 (see Table 1, lower panel), we studied whether we could trigger perceptual reversals by prolonging single frames by either $50 \%$ or $100 \%$. In 312 trials $(13.5 \%)$, perceptual reversals were observed before a switch occurred. As outlined above, these trials were counted as spontaneously generated. The median percept duration of all conditions in Experiment 2 was shorter than in Experiment 1 ( 2.39 vs. $2.69 \mathrm{sec})$. Only in the 5 -fps condition did the switches result in a significant shortening of the percept duration (Friedman rank analysis, $p<.001$ ). The longer switch $(200 \mathrm{msec})$ resulted in the shortest percept durations - that is, in the highest impact on percept reversals (median percept duration: no switch, $2.85 \mathrm{sec}$; 100-msec switch, $2.48 \mathrm{sec}$; 200-msec switch, $2.27 \mathrm{sec}$; Wilcoxon pairwise rank analysis, $p<.001)$. Both the short and long switches (from stimulus onset to the end of the switch frame) correlated significantly with the percept reversal (i.e., percept duration; Spearman's $\rho=.328, p<$ $.001)$, further validating the effect of the manipulation. In the 6- and 7.5-fps conditions, no significant effect of the switch could be observed, although the manipulation did result in a small shortening of percept durations.

\section{DISCUSSION}

This study aimed at two goals. In the first experiment, we studied the relation between the stimulus frequency and the reversal rate, and therefore systematically varied the stimulus frequency and analyzed the resulting percept duration. In the second experiment, we studied whether an irregularity in the presentation sequence could trigger reversals otherwise identical to spontaneous percept reversals. We therefore inserted "switch" events; that is, we elongated the duration of a single frame by either $50 \%$ or $100 \%$.

In Experiment 1, we showed that the percept duration, and with it the reversal rate, significantly depended on the stimulus frequency. Increasing stimulus frequency decreased the duration of perceptual phases (i.e., it increased the spontaneous reversal rate of the illusory rotating circle). The median stable percept duration over all stimulus frequencies was $2.69 \mathrm{sec}$, which is within the range of typical ambiguous figures, such as the Necker cube and those in other apparent-motion paradigms (Gomez, Argandona, Solier, Angulo, \& Vazquez, 1995; Orbach, Zucker, \& Olson, 1966). The robust effect of the stimulus frequency on the reversal rate confirmed the dependence of the percept on primary stimulus properties, such as intensity (Lynn, 1961) and continuity of presentation (Leopold et al., 2002; Orbach et al., 1963). Recently, Brouwer and van Ee (2006) showed that increasing dot density and velocity decreased percept durations in a structure-by-motion paradigm. These effects of exogenous stimulus properties on the reversal rate suggest that perceptual reversals of ambiguous stimuli are influenced, at least, by bottom-up effects on early processing levels. One could argue that eye movements could have triggered the perceptual reversals, but our subjects were intensively trained to focus on the central fixation point and not to track single points of the rotating circle. The response data of single subjects show a very circumscribed mean percept duration, indicating that the reversal did not depend on incidental saccades. In addition, van Dam and van Ee (2006) specifically studied this question, and they demonstrated that saccades correlated with perceptual reversals but did not induce them. Verstraten, Hooge, Culham, and 
Van Wezel (2001) showed basically the same result for the perception of apparent motion, as did Brouwer and van Ee (2006) for the perception of ambiguous spheres.

In Experiment 2, we tried to externally trigger perceptual reversals by prolonging single frames. Since behavioral studies with ambiguous stimuli rely on subjective responses, the efficacy of the manipulation could only be measured by comparing percept durations in the manipulated and unmanipulated conditions. We could not distinguish whether perceptual reversals indicated immediately after a switch were spontaneously generated by coincidence or were indeed externally triggered. To allow for statistical differentiation of the experimental conditions, we inserted the switch frames before the expected spontaneous percept reversal (i.e., mean value of percept duration) and predicted that this should result in an earlier percept reversal, and indeed, this manipulation did result in shorter percept durations. Percept reversals occurred a median of $0.5 \mathrm{sec}$ after the end of the switch, which is in the range of the results of Kanai et al. (2005), who studied the influence of a flash that was presented near different ambiguous stimuli and found that percept reversals typically occurred between 0.5 and $1.0 \mathrm{sec}$ (median) after the flash.

In the 7.5- and 6-fps conditions, no significant effect of the switch was observed. Since the manipulation consisted of a relative prolongation of the presented frames, the absolute duration of the manipulation was shorter in the higher frequencies and may not have sufficed to interfere with the perceived percept. Moreover, higher frequencies resulted in shorter overall percept durations and, hence, in higher temporal overlap of spontaneous and externally triggered reversals (as shown in Experiment 1). Both of these explanations are in line with the fact that the difference between the two manipulations (i.e., $50 \%$ vs. $100 \%$ prolongation of frame presentation) increased with decreasing stimulus frequency. Nevertheless, the results of the 5-fps condition clearly show that a perceptual reversal can be externally triggered by an irregularity in the presentation sequence. Hence, this paradigm will allow for contrasts of externally triggered and (otherwise identical) spontaneously generated perceptual reversals using imaging and electrophysiological techniques and, by this means, for a focus on the underlying mechanism of spontaneous percept reversals.

With respect to the "top-down" versus "bottom-up" debate, our data do not definitely support either interpretation, but rather support a mixed model. Following common argumentation, the effect of the stimulus frequency on the percept duration confirms exogenous (i.e., bottomup) influences of stimulus properties on percept generation. Nevertheless, our observed differences in percept durations cannot solely be attributed to the different stimulus frequencies, since the median durations did not correlate with the number of presented frames (i.e., at lower frequencies, it took significantly fewer frames to elicit a reversal than at higher frequencies). In contrast, considering the relatively large differences between the studied frequencies (i.e., $50 \%$ ), the mean percept durations were relatively stable, suggesting a relatively constant tempo- ral integration of the presented information. We therefore propose that spontaneous percept reversals are primarily based on stimulus-independent, presemantic temporal properties of the brain (Pöppel, 2004), which nonetheless are externally influenced. In the same vein, the effect of the "switch" manipulation should not necessarily be interpreted in favor of a bottom-up mechanism. We suppose that the irregularity in the stimulus frequency caused a breakdown of (otherwise autonomous) integration mechanisms at early processing levels and, in this way, led to the generation of a new percept. To conclude, our paradigm does not definitely support a single interpretation but will allow for specific contrasts of spontaneous and externally triggered (but otherwise identical) perceptual reversals and, by this means, enable further study of this question.

\section{AUTHOR NOTE}

The work was supported by the Gerhard und Irmgard Schulz Fond. We thank Ernst Pöppel (Institute of Medical Psychology, LudwigMaximilians-Universität, München) for reading and commenting on an earlier version of the manuscript. Correspondence related to this article may be sent to R. Ilg, Department of Neurology, Klinikum Rechts der Isar, Technische Universität München, Ismaninger Str. 22, 81675 München, Germany (e-mail: ilg@neuro.med.tum.de).

\section{REFERENCES}

Babich, S., \& Standing, L. (1981). Satiation effects with reversible figures. Perceptual \& Motor Skills, 52, 203-210.

BEER, J. (1989). Learning effects while passively viewing the Necker cube. Perceptual \& Motor Skills, 69, 1391-1394.

Blake, R., \& Logothetis, N. K. (2002). Visual competition. Nature Reviews Neuroscience, 3, 13-21.

Blake, R., Sobel, K. V., \& Gilroy, L. A. (2003). Visual motion retards alternations between conflicting perceptual interpretations. Neuron, 39, $869-878$

Borsellino, A., Carlini, F., Riani, M., Tuccio, M. T., De Marco, A., Penengo, P., \& Trabucco, A. (1982). Effects of visual angle on perspective reversal for ambiguous patterns. Perception, 11, 263-273.

BROUWER, G. J., \& VAN Ee, R. (2006). Endogenous influences on perceptual bistability depend on exogenous stimulus characteristics. Vision Research, 46, 3393-3402.

Bruner, J. S., Busiek, R. D., \& Minturn, A. L. (1952). Assimilation in the immediate reproduction of visually perceived figures. Journal of Experimental Psychology, 44, 151-155.

Bugelski, B. R., \& Alampay, D. A. (1961). The role of frequency in developing perceptual sets. Canadian Journal of Psychology, 15, 205-211.

Cavanagh, P. (1992). Attention-based motion perception. Science, 257, $1563-1565$.

Culham, J. C., Verstraten, F. A. J., Ashida, H., \& Cavanagh, P. (2000). Independent aftereffects of attention and motion. Neuron, 28, 607-615.

Gomez, C., Argandona, E. D., Solier, R. G., Angulo, J. C., \& VAzQueZ, M. (1995). Timing and competition in networks representing ambiguous figures. Brain \& Cognition, 29, 103-114.

Hochberg, J., \& Peterson, M. A. (1987). Piecemeal organization and cognitive components in object perception: Perceptually coupled responses to moving objects. Journal of Experimental Psychology: General, 116, 370-380.

Hol, K., Koene, A., \& van Ee, R. (2003). Attention-biased multi-stable surface perception in three-dimensional structure-from-motion. Journal of Vision, 3, 486-498.

Kanai, R., Moradi, F., Shimojo, S., \& Verstraten, F. A. J. (2005). Perceptual alternation induced by visual transients. Perception, 34, 803-822.

Kleinschmidt, A., Büchel, C., Zeki, S., \& Frackowiak, R. S. J. (1998). Human brain activity during spontaneously reversing perception of ambiguous figures. Proceedings of the Royal Society B, 265, 2427-2433. 
Leopold, D. A., \& Logothetis, N. K. (1999). Multistable phenomena: Changing views in perception. Trends in Cognitive Sciences, $\mathbf{3}$, 254-264.

Leopold, D. A., Wilke, M., Maier, A., \& Logothetis, N. K. (2002). Stable perception of visually ambiguous patterns. Nature Neuroscience, 5, 605-609.

Logothetis, N. K., \& Schall, J. D. (1989). Neuronal correlates of subjective visual perception. Science, 245, 761-763.

Long, G. M., \& OlszWEsKi, A. D. (1999). To reverse or not to reverse: When is an ambiguous figure not ambiguous? American Journal of Psychology, 112, 41-71.

Long, G. M., \& Toppino, T. C. (2004). Enduring interest in perceptual ambiguity: Alternating views of reversible figures. Psychological Bulletin, 130, 748-768.

Long, G. M., Toppino, T. C., \& Kostenbauder, J. F. (1983). As the cube turns: Evidence for two processes in the perception of a dynamic reversible figure. Perception \& Psychophysics, 34, 29-38.

Long, G. M., Toppino, T. C., \& Mondin, G. W. (1992). Prime time: Fatigue and set effects in the perception of reversible figures. Perception \& Psychophysics, 52, 609-616.

Lumer, E. D., Friston, K. J., \& ReEs, G. (1998). Neural correlates of perceptual rivalry in the human brain. Science, 280, 1930-1934.

Lumer, E. D., \& ReEs, G. (1999). Covariation of activity in visual and prefrontal cortex associated with subjective visual perception. Proceedings of the National Academy of Sciences, 96, 1669-1673.

LYNN, R. (1961). Reversible perspective as a function of stimulus-intensity. American Journal of Psychology, 74, 131-133.

Orbach, J., Ehrlich, D., \& Heath, H. A. (1963). Reversibility of the Necker cube: I. An examination of the concept of "satiation of orientation." Perceptual \& Motor Skills, 17, 439-458.

Orbach, J., Zucker, E., \& Olson, R. (1966). Reversibility of the Necker cube: VII. Reversal rate as a function of figure-on and figureoff durations. Perceptual \& Motor Skills, 22, 615-618.

Petersik, J. T., Shepard, A., \& Malsch, R. (1984). A three-dimensional motion aftereffect produced by prolonged adaptation to a rotation simulation. Perception, 13, 489-497.

Peterson, M. A., \& Hochberg, J. (1983). Opposed-set measurement procedure: A quantitative analysis of the role of local cues and intention in form perception. Journal of Experimental Psychology: Human Perception \& Performance, 9, 183-193.

PöPPEL, E. (2004). Lost in time: A historical frame, elementary processing units and the 3-second window. Acta Neurobiologiae Experimentalis, 64, 295-301.

REISBERG, D. (1983). General mental resources and perceptual judgments. Journal of Experimental Psychology: Human Perception \& Performance, 9, 966-979.
Reisberg, D., \& O'Shaughnessy, M. (1984). Diverting subjects' concentration slows figural reversals. Perception, 13, 461-468.

Rock, I., Hall, S., \& Davis, J. (1994). Why do ambiguous figures reverse? Acta Psychologica, 87, 33-57.

Rock, I., \& Mitchener, K. (1992). Further evidence of failure of reversal of ambiguous figures by uninformed subjects. Perception, 21, 39-45.

Slotnick, S. D., \& Yantis, S. (2005). Common neural substrates for the control and effects of visual attention and perceptual bistability. Cognitive Brain Research, 24, 97-108.

Sterzer, P., \& Kleinschmidt, A. (2007). A neural basis for inference in perceptual ambiguity. Proceedings of the National Academy of Sciences, 104, 323-328.

Sterzer, P., Russ, M. O., Preibisch, C., \& Kleinschmidt, A. (2002). Neural correlates of spontaneous direction reversals in ambiguous apparent visual motion. NeuroImage, 15, 908-916.

TopPINO, T. C. (2003). Reversible-figure perception: Mechanisms of intentional control. Perception \& Psychophysics, 65, 1285-1295.

Toppino, T. C., \& LonG, G. M. (1987). Selective adaptation with reversible figures: Don't change that channel. Perception \& Psychophysics, 42, 37-48.

VAN DAM, L. C. J., \& VAN EE, R. (2006). The role of saccades in exerting voluntary control in perceptual and binocular rivalry. Vision Research, 46, 787-799.

Verstraten, F. A. J., \& Ashida, H. (2005). Attention-based motion perception and motion adaptation: What does attention contribute? Vision Research, 45, 1313-1319.

Verstraten, F. A. J., Cavanagh, P., \& Labianca, A. T. (2000). Limits of attentive tracking reveal temporal properties of attention. Vision Research, 40, 3651-3664.

Verstraten, F. A. J., Hooge, I. T., Culham, J., \& Van Wezel, R. J. (2001). Systematic eye movements do not account for the perception of motion during attentive tracking. Vision Research, 41, 3505-3511.

Wertheimer, M. (1912). Experimentelle Studien über das Sehen von Bewegung. Zeitschrift für Psychologie \& Physiologie der Sinnesorgane, 61, 161-265.

Wilms, M., Eickhoff, S. B., Specht, K., Amunts, K., Shah, N. J., Malikovic, A., \& Fink, G. R. (2005). Human V5/MT + : Comparison of functional and cytoarchitectonic data. Anatomy \& Embryology, 210, 485-495.

WOHLSCHLÄGER, A. (2000). Visual motion priming by invisible actions. Vision Research, 40, 925-930.

(Manuscript received September 5, 2006; revision accepted for publication October 3, 2007.) 